\title{
Preparation and In Vitro Evaluation of Self-Emulsifying Drug Delivery System for Oral Delivery of Glibenclamide Akiladevi $D^{* 1}$, Nappinnai $M^{2}$, Jerad Suresh $A^{3}$, Amudha $P^{4}$, Vetrichelvan $T^{5}$
}

\begin{abstract}
${ }^{1}$ Department of Pharmaceutics, Adhiparasakthi College of Pharmacy, Melmaruvathur-603319, Tamilnadu, India
${ }^{2}$ Department of Pharmaceutics, Surya Group of Institutions, School of Pharmacy, Vikravandi, Villupuram-605 652, Tamilnadu, India

${ }^{3}$ Department of Pharmaceutical Chemistry, College of Pharmacy, Madras Medical College, Chennai-600 003, Tamilnadu, India

${ }^{4}$ Department of Pharmacology, CL Baid Mehtha College of Pharmacy, Thorapakkam, Chennai, Tamilnadu, India

${ }^{5}$ Department of Pharmaceutical Analysis, Adhiparasakthi College of Pharmacy, Melmaruvathur-603 319, Tamilnadu, India
\end{abstract}

*Corresponding author: Akiladevi D, Department of Pharmaceutics, Adhiparasakthi College of Pharmacy, Melmaruvathur-603319, Tamilnadu, India, E-mail: akilaajcp@gmail.com

Received: February 28, 2017; Revised: March 17, 2017; Published: March 21, 2017

Copyright: @2017 Akiladevi D, et al. This is an open-access article distributed under the terms of the Creative Commons Attribution License, which permits unrestricted use, distribution, and reproduction in any medium, provided the original author and source are credited. The article has been previewed and authenticated by the Authors before sending the publication for print. The Journal, Editor and the Editorial Board are not entitled or liable to either justify or responsible for inaccurate and misleading data if any. It is the sole responsibility of the Author concerned.

Citation: Akiladevi D, Nappinnai M, Jerad Suresh A, et al. Preparation and In Vitro Evaluation of Self-Emulsifying Drug Delivery System for Oral Delivery of Glibenclamide. Int J Pharm Pharmacol 2017; 1: 105.

\footnotetext{
Abstract

Objective: The present study was aimed to develop a novel o/w self-emulsifying drug delivery system (SEDDS)with novel synthetic oils, a variety of natural oils and surfactants/co surfactants to enhance the solubility and dissolution rate of poorly water soluble drug, glibenclamide thereby to improve its relative bioavailability. The novelty in this proposed research work is the simple process of production and easy to scale up with minimum infrastructure.

Methods: The design of an optimal SEDDS is performed by preformulation solubility and ternary phase-diagram studies. The prepared self - emulsifying formulations were performed for in vitro evaluation studies of droplet size, polydispersity index, drug loading, zeta potential, optical clarity, turbidity, cloud point, viscosity determination, self-emulsification time assessment, in vitro drug release and in vitro diffusion studies.

Results: The results showed that Peceol as oil, Labrasol as surfactant and Transcutol HP as cosurfactant prepared stable emulsions has a refractive index as same that of water clearly indicated that the microemulsions was highly transparent. The percentage drug release for optimal formulation of glibenclamide SEDDS after 30 minutes was found to be $99.7 \%$ when compared to API (active pharmaceutical ingredient) and marketed tablet.

Conclusion: This study demonstrated that in vitro drug release and in vitro diffusion study were dependent on critical parameters such as particle size and drug loading in SEDDS formulations

Keywords: Self -Emulsifying Drug Delivery, Glibenclamide, In vitro evaluation, Particle size, Drug loading
} 
The major, popular route of administering drug from time immemorial is the oral route for both chronic and acute dosage regimen. Unfortunately, more than $50 \%$ of drug compounds have unfavourable physicochemical property of which high lipophilicity is major one. Almost $40 \%$ of the new drug candidates exhibit low solubility in water which leads to poor bioavailability, high intra and inter -subject variability, lack of dose proportionality. Thus, for such compounds, among numerous factors limiting bioavailability is the absorption rate from gastrointestinal lumen. This is in turn is related to dissolution [1]. According to the BCS classification, two classes of drugs show poor aqueous solubility namely BCS II and BCS IV. BCS II drugs possess poor aqueous solubility but have good permeation properties. BCS class IV drugs are poorly water soluble and poorly permeable. The enhancement of oral bioavailability of poorly water soluble drugs remains one of the most challenging aspects of drug development. Although salt formation, solubilization and particle size reduction have commonly been used to increase dissolution rate, there are practical limitations of these techniques. The salt formation is not feasible for neutral compounds and the synthesis of appropriate salt forms of drugs that are weakly acidic or weakly basic may often not be practical. The solubilization of drugs in organic solvents or in aqueous media by the use of surfactants and co-solvents leads to liquid formulations that are usually undesirable from the viewpoints of patient acceptability and commercialization. Although particle size reduction is commonly used to increase dissolution rate, there is a practical limit to how much size reduction can be achieved by such commonly used methods as controlled crystallization, grinding, etc. The use of very fine powders in a dosage form may also be problematic because of handling difficulties and poor wettability [2]. To overcome these drawbacks, various formulation strategies have been adopted which including the use of cyclodextrins, nanoparticles, solid dispersions, and permeation enhancers [3]. SEDDS are considered one of the promising approaches for tackling formulation problems associated with drugs with poor aqueous solubility. SEDDS is Akiladevi D, et al. Int J Pharm Pharmacol an oral lipid dosage form composed of a mixture of natural or synthetic oils, solid or liquid surfactants, or with one or more solvents of hydrophilic capacity and co solvents/surfactants [4]. These formulations disperse freely when they come to contact with gastric fluids and form an $\mathrm{o} / \mathrm{w}$ emulsion or micro emulsion utilizing mild agitation conditions provided by gastric motility. The lipophilic drug is delivered in liquid form, in small droplets of oil, leading to the elimination of the dissolution which is rate limited process in the absorption of poorly soluble drugs. The bioavailability of SEDDS is thereby improved and the drug content in plasma profile is reproducible in such systems.

\section{Advantages of the system [5]}

$>$ The drug absorption will be more: SEDDS formulations can enhance the bioavailability by increasing the solubility of the drug and minimizes the gastric irritation. In SEDDS, the lipid phase interacts readily with water, forming a fine particulate $\mathrm{o} / \mathrm{w}$ emulsion. The emulsion droplets will deliver the drug to the gastrointestinal mucosa in the dissolved state readily accessible for absorption.

$>$ The drug can be protected from the gut environment: Many drugs are degraded in the physiological system because of acidic $\mathrm{pH}$ in the stomach, enzymatic degradation or hydrolytic degradation. Such drugs, when presented in the form of SEDDS, can be well protected against the degradation processes as a liquid crystalline phase in SEDDS might act as the barrier between the degrading environment and the drug [6].

$>$ The sensitive drug compounds are protected: The self-micro emulsifying formulations spread readily in the GI tract and the digestive motility of the stomach and the intestine provide the agitation necessary for self-emulsification. SEDDS typically produce an emulsion with a droplet size between 100 and $300 \mathrm{~nm}$ while SMEDDS form a transparent micro emulsion with a droplet size of less than 50 $\mathrm{nm}$. When compared with emulsions which are sensitive and metastable dispersed forms, SEDDS and SMEDDS are 
physically stable formulations that are easy to manufacture [7].

$>$ The oral dose is reduced therefore, the oral bioavailability is enhanced.

Glibenclamide (GBD) a second-generation sulfonylurea is orally used as a hypoglycemic agent to treat noninsulin-dependent (type II) diabetes mellitus. The aqueous solubility of GBD is low and highly $\mathrm{pH}$-dependent in the physiological range because of its $\mathrm{pKa}$ value of 5.3. Low aqueous solubility gives rise to unsatisfactory dissolution profiles leading to potential problems of poor bioavailability and bioequivalence of the drug's dosage form GBD. The low oral dose $(5-15 \mathrm{mg})$, suitable $\log \mathrm{p}$ (octanol/water) of 4.8 , and it being a BCS class II drug strongly provide a rationale to develop an SEDDS of GBD. In our study glibenclamide SEDDS was prepared and in vitro evaluation studies were performed to improve the solubility and dissolution rate.

\section{Materials and Methods}

\section{Materials}

Glibenclamide calcium was generously gifted by Goodman Pharmaceuticals, Pondicherry. Capryol PGMCtranscutol HP, peceol, labrasol, labrafil M 1944 CS and labrafil M 2125 CS were gift samples from Gattefosse, Mumbai. Virgin sesame oil, virgin coconut oil and sunflower oil were obtained from Vama oil industries, Coimbatore, Olive oil from Shaah Enterprises, Chennai, Mustard oil from Green spice products, Coimbatore, Rice bran oil from Jupiter Manufacturing industry, Chennai, Corn oil from Arumuga group of industries, Tamilnadu were obtained for the research work. The dialysis membrane of molecular weight cut off 12000 daltons was purchased from Himedia Pvt. Ltd. All other chemicals were of analytical grade.

\section{Methods}

\section{Fourier Transform Infrared (FT-IR) Spectroscopy of Glibenclamide [8]}

The drug sample was mixed with anhydrous potassium bromide (KBr) in 1:4 ratios. Briefly about $100 \mathrm{mg}$ of this mixture was made into fine powder using mortar and pestle followed by compression to form transparent $\mathrm{KBr}$ pellet using Techno search hydraulic press set at 15-ton pressure. Each $\mathrm{KBr}$ pellet was scanned at $4 \mathrm{~mm} / \mathrm{s}$ at a resolution of $2 \mathrm{~cm}$ over a wave number region from 4000 to $400 \mathrm{~cm}^{-1}$ in an FTIR spectrophotometer (8400S Shimadzu, Japan). Figure 1 shows FTIR spectrum of pure drug.

\section{Solubility Studies}

The solubility of glibenclamide was determined in distilled water, methanol, ethanol and dichloromethane. About $2 \mathrm{ml}$ of each solvent was transferred into $5 \mathrm{ml}$ glass vial and an excess quantity of drug $(150 \mathrm{mg})$ was added to the vial. The solubility of the drug samples was also analyzed by adding excess amount $(150 \mathrm{mg})$ of the drug to $2 \mathrm{ml}$ of various oils, surfactants, and co-surfactants in screw capped glass vials followed by vortex mixing for 30 sec using vortex mixer (Sphinx, Japan). The mixtures were shaken for $48 \mathrm{~h}$ at $30^{\circ} \mathrm{C}$ in a thermostatically controlled shaking water bath, followed by equilibrium for $24 \mathrm{hr}$. The sample mixtures were then centrifuged at $3000 \mathrm{rpm}$ [9] for $10 \mathrm{~min}$ and the supernatant liquid was filtered through a millipore membrane filter $(0.45 \mu)$. Samples were suitably diluted with methanol followed by sonication for $10 \mathrm{~min}$ and finally diluted with the same solvent. The final drug concentration was quantified by UVvisible spectrophotometer at $226.5 \mathrm{~nm}$ for glibenclamide. The experiment was repeated in triplicates. The results are represented as mean value $(\mathrm{mg} / \mathrm{ml}) \pm \mathrm{SD}$.

\section{Construction of Ternary Phase Diagram}

The percentage limit of surfactant, cosurfactant and oil used was selected by considering their acceptable safe dose and decided on the basis of the requirements stated according to the lipid formulation classification system (LFCS) introduced by Pouton. A ternary phase diagram was constructed for the system containing oil-surfactant-co-surfactant by Chemix School software version 3.51. A series of self-emulsifying systems were prepared with varying weight percentage of oil, surfactant, and co-surfactant. Since the drug incorporated in the SEDDS may have some effect on self-emulsion boundary, every system in the series also consisted of $5 \% \mathrm{w} / \mathrm{w}$ for 
glibenclamide. These mixtures were diluted dropwise with double distilled water with moderate agitation.

\section{Preparation of SEDDS}

A series of SEDDS formulations were prepared using labrasol and transcutol hp as the S/CoS combination and Peceol as the oil based on solubility studies as described in Table 1. In all the formulations, the level of glibenclamide was kept constant (5 mg). Briefly, accurately weighed glibenclamide was placed in a glass vial, and oil, surfactant, and cosurfactant were added. Then the components were mixed by gentle vortexing and sonicated for 10 minutes. Altogether nine formulations were prepared as shown by Table 2 .

\section{Evaluation of Prepared SEDDS}

\section{Self-Emulsification and Drug Precipitation Studies}

The efficiency of self-emulsification of oral micro/nano-emulsion is assessed by dispersibility test using a standard USP dissolution apparatus II [10]. One $\mathrm{ml}$ of each formulation is added to $500 \mathrm{ml}$ of water at $37 \pm$ $0.5^{\circ} \mathrm{C}$. A standard stainless steel dissolution paddle rotating at $50 \mathrm{rpm}$ tends to provide gentle agitation. The in vitro performance of the formulations is visually assessed from such dispersion, using a suitable grading system. The grading system has been reported to be based on the formation of a micro emulsion (o/w or w/o), micro emulsion gel, emulsion or emulgel. The drug/excipient precipitation was evaluated by visual inspection of the resultant emulsion after $24 \mathrm{~h}$ (Table 3 ). The in vitro performance of the formulations is visually assessed using the following grading system:

Grade I: Rapidly forming (within $1 \mathrm{~min}$ ) nano emulsion, having a clear or bluish appearance. (Micro emulsion)

Grade II: Rapidly forming, slightly less clear emulsion, having a bluish white appearance. (Micro emulsion gel)

Grade III: Fine milky emulsion that formed within 2 min. (Emulsion)

Grade IV: A dull grayish white emulsion having slightly oily appearance that is slow to emulsify is formed (longer than $2 \mathrm{~min}$ ) (Emulgel).
Grade V: Formulation, exhibiting either poor or minimal emulsification with large oil globules present on the surface with phase separation is observed.

Grade VI: The drug is precipitated.

Grade I and Grade II formulation will remain as nano-emulsion when dispersed in GIT. The formulation falling in Grade III could be recommended for SEDDS formulation. The primary means of self-emulsification is a visual evaluation. The efficiency of selfemulsification could be estimated by determining the rate of emulsification, droplet size distribution, and turbidity measurements.

\section{Phase Separation Study}

The self-emulsifying formulation was diluted with distilled water up to 5 times and the temperature was maintained at $25^{\circ} \mathrm{C}$. The mixture was then mixed for $2 \mathrm{~min}$, stored for about $2 \mathrm{~h}$ and visually observed for any phase separation.

\section{Determination of Emulsification Time}

The emulsification time (the time for a preconcentrate to form a homogeneous mixture upon dilution) was monitored by visually observing the disappearance of SEDDS and the final appearance of the emulsion in triplicate. A dissolution apparatus USP II (Electrolab) was employed with $500 \mathrm{ml}$ water and with a paddle speed of $50 \mathrm{rpm}$ at $37^{\circ} \mathrm{C}$. The SEDDS $(1 \mathrm{ml})$ was added drop wise to the medium by dropping the pipette and time required for the disappearance of SEDDS was recorded [11].

\section{Spectroscopic Characterization of Optical Clarity}

SEDDS formulations disperse in aqueous phase forming the emulsion or micro emulsions and can be detected by the final appearance and droplet size. In practice, the key difference between the emulsion and micro emulsions concerns with their appearance. Emulsions are cloudy while micro emulsions are clear or translucent and the reason for their transparency appearance is due to very small droplet size. The optical clarity may be checked visually. But in order to measure it quantitatively, a UV-visible spectrophotometer was used to measure the amount of light of a 
given wavelength absorbed by the solution. The cloudier solutions will absorb more of the incident light, resulting in higher absorbance values and lower absorbance is obtained with optically clear solutions.

The optical clarity of aqueous dispersions of SEDDS formulations was measured spectroscopically. About $1 \mathrm{ml}$ of SEDDS formulations were diluted to 50 times with double distilled water. The absorbance values of each formulation were measured by a UV-visible spectrophotometer (Shimadzu) at $400 \mathrm{~nm}[12]$.

\section{Turbidity Measurement}

The measurement of turbidity is to analyze whether the dispersion reaches equilibrium rapidly and in a reproducible time. The growth of emulsification is done by nepheloturbidimetric evaluation. The turbidity measurements in nephelometric turbidity unit (NTU) were performed on the resultant emulsion stored in a screw capped sample vials using a turbid meter (Elico D-10-model 331). $0.5 \mathrm{ml}$ of the SEDDS formulation was introduced into $250 \mathrm{ml}$ of distilled water in 500 $\mathrm{ml}$ conical flask under an action of magnetic stirrer rotating at constant speed. The emulsification was done at room temperature [13].

\section{Viscosity Determination}

The viscosity studies are necessary for SEDDS to characterize the system physically and to control its stability. If the system has low viscosity then, it is o/w type of the system and if a high viscosity then it is w/o type of the system. SEDDS preconcentrate $(10 \mathrm{ml})$ was taken and its viscosity was measured by using Brookfield viscometer (Brookfield engineering Laboratories, USA) using spindle C 16-1 at 25 $\pm 0.5^{\circ} \mathrm{C}$ with a shear rate of $50 \mathrm{rpm}$.

\section{Cloud Point Measurement}

Cloud point temperatures (Tc) was determined by visual observation. $0.5 \mathrm{ml}$ of preconcentrate (Oil-20 mg and Smix-40 mg) was diluted to $50 \mathrm{ml}$ with distilling water in a glass beaker. The sample was heated at the rate of about $0.5^{\circ} \mathrm{C} / \mathrm{min}$. A close observation was made at the appearance of the dispersion with the increase in temperature. The temperature at which the dispersion became turbid was taken as Tc. After the temperature exceeds the cloud point, the sample was cooled below Tc, and then it was heated again to check the reproducibility of the measurements. It mainly insists about the stability of micro emulsion at body temperature [14].

\section{Determination of Refractive Index}

The refractive index, $\mathrm{n}$, of a medium is defined as the ratio of the speed, c, of a wave such as light or sound in a reference medium to the phase speed, $v p$, of the wave in the medium represented by $n=c / v p$. It was determined using an Abbes type refractometer. The clarity of micro emulsion could be estimated by measuring the refractive index of the formulations [1517]. The SEDDS formulations were diluted 100 times with water. The refractive index of the system was measured by an Abbe refractometer by placing 1 drop of solution on the slide and it compares with distilled water. The refractive index (RI) was determined to prove the isotropicity of the developed SEDDS formulations close to that of the water (1.333). The closure of the formulations RI value to water indicated the transparency property of the formulations.

\section{Droplet Size and Polydispersity Index (PDI) Analysis}

The droplet size of the micro/nano emulsions is determined by photon correlation spectroscopy (which analyses the fluctuations in light scattering due to a brownian motion of the particles) using a Zetasizer which can measure sizes between 10 and $5000 \mathrm{~nm}$. Polydispersity was determined according to the equation:

Polydispersity = D (0.9) $-\mathrm{D}(\mathbf{0 . 1}) / \mathrm{D}(\mathbf{0 . 5})$

Where D (0.9) corresponds to particle size immediately above $90 \%$ of the sample, D (0.5) corresponds to particle size immediately above $5 \%$ of the sample, and D (0.1) corresponds to particle size immediately above $10 \%$ of the sample. PDI is a measure of particle homogeneity and it varies from 0.0 to 1.0 . The closer to zero the PDI value the more homogenous are the particles. The mean droplet size and polydispersity index of formulations were determined by using 
Malvern Nano Zeta sizer-90. The resultant SEDDS $0.5 \mathrm{ml}$, was diluted to $100 \mathrm{ml}$ with double distilled water. The samples were loaded into a cuvette placed in a thermostatic chamber and light scattering was monitored at $25^{\circ} \mathrm{C}$ at a $90^{\circ}$ angle [16] after external standardization with spherical polystyrene beads.

Each determination was done in triplicate. The nanometric size range of the particle is retained even after 100 times dilution with water which proves the systems compatibility with excess water.

\section{Zeta Potential Measurement}

The zeta potential of prepared SEDDS formulations was determined using a Zeta sizer ZS 90 (Malvern Instruments UK) by using laser Doppler micro-electrophoresis. An electric field is applied to a solution which will cause the particles to move to the electrodes with a velocity related to their zeta potential calculated using Helmholtz-Smoluchowski equation. A suitable amount of the sample (50$100 \mu \mathrm{l}$ ) was diluted with $5 \mathrm{ml}$ of distilled water and after sonicating in a bath sonicator to achieve a homogeneous state. Measurements were carried out at $25^{\circ} \mathrm{C}$ using disposable polystyrene cuvette with a zeta dip cell. All the measurements were performed in triplicate and the data presented is mean \pm SD [1717].

Zeta potential determination using following equation

$\zeta=\frac{V}{E} \cdot \frac{\eta}{\varepsilon \cdot \varepsilon 0}$

$\zeta$ zeta potential, E electrical intensity, v particle velocity, $\eta$ viscosity, $\varepsilon \cdot \varepsilon 0$ dielectric constant

\section{Drug Loading Efficiency [18]}

The drug efficiency was done to investigate the effect of drugs on a self-emulsifying performance of SEDDS. Approximately $10 \mathrm{mg}$ of glibenclamide was added to $1 \mathrm{ml}$ of boundary formulations of SEDDS and checked for a formation of the clear solution. The prototype formulations of glibenclamide were prepared by varying peceol in 1:1 ratios of the mixture of labrasol and transcutol HP as per the formula composition mentioned in Table 2. In the first trial, the oil was used at $15 \%$ with an interval of $5 \%$ and increased up to $25 \%$. The ratio of surfactant to co-surfactant was maintained at $1: 1$. Then drug of one dose equivalent of $5 \mathrm{mg}$ of glibenclamide was added and stirred for $15 \mathrm{~min}$. The mixture was heated to $30-40^{\circ} \mathrm{C}$ till the drug was solubilized. The drug loading capacity of each mixture was determined by adding the excess of glibenclamide to each prototype mixture till the clear solution was obtained. The solution was filtered using a membrane filter $(0.45 \mu \mathrm{m}, 13$ $\mathrm{mm}$, Whatman filter).

The drug content of the SEDDS formulation was determined by diluting the solution in methanol and the volume was made up to $10 \mathrm{ml}$ with methanol $(1 \mathrm{mg} / \mathrm{ml})$. From the above stock solution, $0.2 \mathrm{ml}(200 \mu \mathrm{g} / \mathrm{ml})$ was withdrawn and diluted up to $10 \mathrm{ml}$ with methanol $(20 \mu \mathrm{g} / \mathrm{ml})$. From the above solution $0.2 \mathrm{ml}(20 \mu \mathrm{g} / \mathrm{ml})$ diluted up to $10 \mathrm{ml}$ with methanol $(2 \mu \mathrm{g} / \mathrm{ml})$ Samples were prepared in triplicate and absorbance was measured at $226.5 \mathrm{~nm}$ using UV-visible Spectrophotometer [19] (Shimadzu UV-1700) using methanol as a reference solution. The drug content was determined from the standard calibration curve of glibenclamide in methanol using the regression equation $y=0.118 x+0.002$ with the correlation coefficient of 0.999 . The procedure procedure mentioned above with the SEDDS formulation equivalent to $5 \mathrm{mg}$ of glibenclamide and the absorbance was measured at $226.5 \mathrm{~nm}$ using UV-Visible spectrophotometer (Shimadzu UV-1700).

Drug loading efficiency was calculated by equation

Drug loading efficiency $=$ Amount of drug in known amount of formulation $x 100$ / Initial drug load

\section{In Vitro Dissolution Studies of Glibenclamide}

The SEDDS formulation of GF7* were optimized by trial error and method and validated by preparing four formulations with similar composition as that of GF7*. The in vitro evaluation studies were performed and the resulting formulation was considered as best and optimized formulation as the results obtained were with minimum particle size and maximum drug loading for stable formulation of SEDDS which was showed in Table 2 and 
Table 4. The in vitro release rate of four validated formulation were similar to that optimized formulation of GF7* as shown in vitro release graph depicted in Figure 3 . The in vitro drug release profiles of optimized SEDDS formulations of glibenclamide GF7*, API glibenclamide and marketed glibenclamide tablet (Daonil $5 \mathrm{mg}$, Aventis Pharma Ltd) were carried out using USP type II dissolution test apparatus (Electrolab) in $900 \mathrm{ml}$ of phosphate buffer ( $\mathrm{pH} 7.4)$ as dissolution media [20]. The temperature was maintained at $37 \pm 0.5^{\circ} \mathrm{C}$ and the speed of the paddle was set at $50 \mathrm{rpm}$. About $70 \mathrm{mg}$ of each optimized SEDDS formulation $(\mathrm{GF} 7 *$ ) were filled into soft gelatin capsules (size ' 3 ') and used for dissolution studies. The capsules were held to the bottom of the vessel using copper sinkers. At predetermined time intervals of $5,10,15,20$, and $30 \mathrm{~min}$, an aliquot $(2 \mathrm{ml})$ of a sample was collected and filtered through the membrane filter $(0.45 \mu \mathrm{m}$, Whatman). The withdrawn samples were diluted suitably with phosphate buffer ( $\mathrm{pH}$ 7.4) and the drug content was analyzed by standard calibration curve method using the linear regression equation $y=0.018 x+0.001$ of glibenclamide in phosphate buffer $\mathrm{pH} 7.4$ with the correlation co efficient of 0.999 by UV-visible spectrophotometer (Shimadzu UV-1700) at $226.5 \mathrm{~nm}$. An equal volume of the dissolution medium was replaced in the vessel after each withdrawal to maintain the sink condition. Each test was performed in triplicate $(n=3)$, and calculated mean values of cumulative drug release were used while plotting the release curves as illustrated in Figure 3. The dissolution profile of the API glibenclamide and marketed tablet (Daonil 5 $\mathrm{mg}$ ) were assessed by the same method.

\section{In Vitro Diffusion Release Study of Glibenclamide}

The in vitro diffusion was performed using the dialysis membrane diffusion technique by a dialysis membrane method. The dialysis membrane of molecular weight cut off 12000 daltons was soaked in distilled water for four hours and then rinsed thoroughly with distilled water. One end of pretreated cellulose dialysis bag (7 cm tubing) was sealed firmly with clamp and $0.5 \mathrm{ml}$ of optimized selfemulsifying formulation was introduced in it along with $0.5 \mathrm{ml}$ of dialyzing medium Akiladevi D, et al. Int J Pharm Pharmacol (phosphate buffer $\mathrm{pH}$ 7.4). The other end of the bag was also secured with clamp and was allowed to rotate freely. The bags were incubated in beakers containing $500 \mathrm{ml}$ phosphate buffer 7.4 for glibenclamide. $5 \mathrm{ml}$ of samples were withdrawn individually at $0.5,1$, and 2 hours for glibenclamide, which was simultaneously replaced with equal volumes of fresh medium at the same time. The drug content was determined spectrophotometrically from the standard calibration curve of glibenclamide in phosphate buffer $(\mathrm{pH} \mathrm{7.4)}$ as shown in Figure 4 using the linear regression equation $y=0.018 x+0.001$ with the correlation co efficient $\left(\mathrm{r}^{2}\right)$ of 0.9995 at $227.5 \mathrm{~nm} \mathrm{UV}$ visible spectrophotometer (Shimadzu UV1700). The diffusion of the drug from optimized formulation was compared with the API and marketed tablet.

\section{Discussion}

\section{Solubility Study}

Peceol a novel a semi synthetic medium chain derivative was chosen as oil phase for glibenclamide since it was found to have maximum solubility when compared to the other oils and in which the solubility was found to be minimum in sunflower oil as indicated in results of Table 1. Peceol is an efficient solubilizer for glibenclamide which was proven by Patil Prashant $\mathrm{P}$ et al. [21] in his research work. From Table 1 it was proved that labrasol was chosen as surfactant because it showed maximum solubility followed by labrafil 1944 CS, capryol PGMC and labrafil 2125 for formulation of glibenclamide SEDDS. The medium length alkyl chain surfactant labrasol was selected as a surfactant for both the drugs since it has maximum solubilizing capacity and it was represented for drugs having poor intestinal absorption [22]. Transcutol HP showed maximum solubility for glibenclamide and it was selected as the cosurfactant for the formulation of SEDDS as indicated in Table 1. Transcutol HP is a strong solubilizer with low toxicity has a long history of safe use as a solvent in many products including pharmaceuticals, cosmetics and food applications [23].

Construction of Phase diagram 
In the present study peceol (oil) with labrasol and Transcutol $\mathrm{HP}$ as the S/CoS mixture was selected from the solubility studies. From the ternary plot as shown in Figure 2, Peceol gave a wider micro emulsion region at $1: 1$ of Smix ratio for glibenclamide. The co-surfactant was incorporated along with the surfactant in equal proportion at Smix ratio of 1:1 a higher nano-emulsion region was observed in glibenclamide SEDDS. This may be due to the addition of co-surfactant leading to further decrease in the interfacial tension, which will lead to increase in the fluidity of the interfacial film, thus increasing the entropy [24]. The percentage of oil, surfactant and cosurfactant selected for both the drugs were selected from the phase diagram and only those formulations which used the minimum and maximum concentration of Smix were taken for the formulation of SEDDS. Moreover, the self-emulsification ability of SEDDS depends on the formulation parameter variables such as surfactant/cosurfactant and oil ratio, a polarity of the emulsion, globule size and charge on the droplets. The stability and efficiency of the drug is increased by self-emulsification property.

\section{Self-Emulsification, Drug Precipitation, Phase Separation Andassessment of Emulsification Time Studies}

The self-emulsification was visually assessed to measure the apparent spontaneity of nanoemulsion formation. SEDDS when diluted in water were found to be non-turbid and bluish transparent in appearance indicating spontaneous emulsification. All the resulting nanoemulsions were transparent with some opalescence in appearance and did not show any sign of phase separation. All the results of the nanoemulsion formulations were transparent and their absorbance's were below 1 which showed good optical clarity as illustrated in Table 4. The selection of surfactants and cosurfactants are determined by emulsification ability which depends on the physicochemical properties such as globule size, Zeta potential, turbidity measurement and PDI of the resulting nanoemulsion. All the formulations of both the drugs showed rapid emulsification time within a minute which proves the performance of the formulations for enhancing the dissolution profile. Thus, it can Akiladevi D, et al. Int J Pharm Pharmacol be concluded that the absorption of the drug can be increased in vivo if the formulations have low emulsification time. Labrasol a hydrophilic surfactant having HLB value of 12 rendered very good nanoemulsions that required a short emulsification period.

\section{Turbidity Measurements}

The rapid equilibrium reached by the dispersion and reproducibility of the process is determined by turbidity measurements. The results obtained are within the limits for both the drugs. The emulsification efficiency is confirmed by a decrease in turbidity values which results in the corresponding decrease in droplet size. In the formulation GF5 of glibenclamide SEDDS the turbidity value was high of 142 NTU due to the larger droplet size of the emulsion formed of $402.3 \mathrm{~nm}$ which was shown in Table 2 and Table 4.

\section{Refractive Index and Viscosity Measurement}

There was no significant difference in the refractive index values of the formulations tested. The refractive index values close to that of the water (1.333) prove the isotropicity of the formulations as indicated in Table 4.

\section{Droplet Size}

The globule size observed for all the formulation was less than $620 \mathrm{~nm}$. The drug loading did not show significant difference in the polydispersity values. The droplet size distribution is one of the most important characteristics of nano-emulsion for stability evaluation and is a critical step in the pathway of enhancing drug bioavailability. The smaller nano-emulsion particle size leads to larger interfacial surface area, thus promoting rapid absorption and improved bioavailability. The particle size and drug loading are critical formulation parameters used to help maximize the pharmacokinetics of small molecules [25]. In this present work, the particle size of SEDDS after dilution was selected as criteria for in vitro evaluation. If the particle size of SEDDS is less, the release of drug will be more resulting with better bioavailability in the formulation of SEDDS. The particle size of around $20 \mathrm{~nm}$ gives total transparent system upon dilution, which acts as a solution. 


\section{Zeta Potential}

The emulsion stability is directly related to the magnitude of the surface charge and the zeta potential is stability indicative parameter in colloidal systems means the system will resist aggregation. The reason for this behavior could be attributed to the strong repulsive Coulomb force between charged particles, which counterbalances the Vander Waals attraction force. Generally, an increase in electrostatic repulsive forces between micro emulsion droplets prevents the coalescence of micro emulsion droplets. High absolute (positive and negative) zeta potential values (above +30 or $-30 \mathrm{mV}$ ) should preferably be achieved in most of the emulsions prepared in order to ensure the creation of a high-energy barrier against coalescence of the dispersed droplets [26]. The zeta potential of the optimized formulation of glibenclamide was found to be $-24.8 \mathrm{mV}$ which were nearer the limits with good separation. The zeta potential values were found to carry negative charges due to the presence of free fatty acids. Significant increase in the value of zeta potential was observed after drug loading, higher absolute values of zeta potential generally, indicated an increase of electrostatic repulsive forces between emulsion droplets preventing the coalescence droplets and increases in the stability. Among all the vehicles tested Labrasol (surfactant) and Transcutol HP (co-surfactant) proved to be the most promising vehicles for SEDDS formulation.

\section{Polydispersity Index (PDI)}

The PDI for all the formulations were less than 0.5 , formulation with combination of Smix showed lower PDI values as illustrated with the results given in Table 4, thus indicating the uniform size distributionimproving the performance of the spontaneous emulsification. Labrasol is a high dispersible surfactant produces small droplet size and good PDI from all surfactants.

\section{Cloud Point}

The cloud point is an essential factor in the SEDDS consisting of non-ionic surfactants, and it is responsible for the successful formation of a stable microemulsion. When the temperature is higher than the cloud point, an irreversible phase separation will occur and the cloudiness of the preparation would have a bad effect on drug absorption, because of the dehydration of the polyethylene oxide moiety. Hence, the cloud point for SMEDDS should be above $37^{\circ} \mathrm{C}$, which will avoid phase separation occurring in the gastrointestinal tract. The cloud point for all the formulation as shown in Table 4 tested was above $37^{\circ} \mathrm{C}$. Therefore, it would suggest a stable micro emulsion can be formed at physiological temperature in-vivo.

\section{Drug Loading}

The maximum drug loading for glibenclamide ranged from $8.2 \mathrm{mg}$ to $9.23 \mathrm{mg}$ for all nine formulations. Drug loading is a critical parameter which and toxicity of the drug. Higher drug loading is preferable because less non-active excipie affects the therapeutic efficacy, pharmacokinetics nts are used to produce the same quantity of API in the SEDDS formulation. At a higher drug loading, lower quantity of oils and surfactants (nonactive ingredients) need to be manufactured to deliver an equivalent dose of API. The benefit of maximum drug loading is that the quantity of surfactants incorporated in the SEDDS formulation can be reduced which in turn large quantity of the surfactants causes the irritation on GIT. It can also reduce the manufacturing and processing time, raw material usage. So, particle size was selected as criteria for the optimization. The percentage drug loading was selected as another critical parameter for in vitro evaluation because the higher the drug loading in SEDDS formulation reduces final dose of the drug and improves patient compliance with minimum GIT irritation and side effects.

\section{In Vitro Dissolution Study}

The dissolution study of API, marketed formulation and optimized formulation GF7* were performed in $7.4 \mathrm{pH}$ phosphate buffer for glibenclamide. The optimized glibenclamide SEDDS formulation of GF7* showed a drug release of above $90 \%$ within $5 \mathrm{~min}$ was illustrated in Table 5. It could be suggested that the SEDDS formulation resulted in spontaneous formation of a micro emulsion 
with a small droplet size, which permitted a faster rate of drug release into the aqueous phase, much faster than that of plain glibenclamide and marketed tablet as shown in Figure 3. Thus, this greater availability of dissolved glibenclamide from the SEDDS formulation could lead to higher absorption and higher oral bioavailability. It was also showed that increase in surfactant concentration and decrease in oil concentration in formulation increase in drug release.

\section{In Vitro Diffusion Release Study}

Diffusion study was carried out to study the release behavior of formulation from liquid crystalline phase around the droplet using dialysis technique. In vitro diffusion profile of glibenclamide from optimized SEDDS in phosphate buffer ( $\mathrm{pH}$ 7.4) is given in Table 6. It was observed that at the end of $2 \mathrm{~h}$, the optimized formulation GF7* SEDDS showed about $99.8 \%$ and $96.23 \%$ for the marketed tablet (Daonil $5 \mathrm{mg}$ ) at the end of $2 \mathrm{~h}$.

\section{References}

1. Amidon GL, Lennernas $\mathrm{H}$, Shah VP, et al. A theoretical basis for a biopharmaceutic drug classification: the correlation of in vitro drug product dissolution in vivo bioavailability. Pharm Res 1995; 12: 413420.

2. Craig DQM. The use of emulsifying systems as a means of improving drug delivery B.T Gattefosse 1993; 86.

3. Aungst BJ. Novel formulation strategies for improving oral bioavailability of drugs with poor membrane permeation or presystemic metabolism. J Pharm Sci 1993; 82: 979987.

4. Charman SA, Charman WN, Rogge MC, et al. Self-emulsifying drug delivery systems formulation and biopharmaceutic evaluation of an investigational lipophilic compound. Pharm Res 1992; 9: 87-93.

5. Patel PA, Chaulang GM, Akolkotkar A, et al. Self-emulsifying drug delivery systems: A Review. Res J Pharm Technol 2008; 1: 313-323.

6. Nigade PM, Patil S, Tiwari ST. Self emulsifying drug delivery system: A Review. Int J Pharm Bio Sci 2012; 2: 42-52
7. Kamble AV, Jagdale DM, Kadam VJ. SelfMicro Emulsifying Drug Delivery System. Int J Pharm Bio Sci 2010; 2: 2-9.

8. Indian Pharmacopoeia Vol II; 2007: 547548

9. Bora DK, Borude P, Bhise K. Formulation and Evaluation of Self-microemulsifying drug delivery system of low solubility drug for enhanced solubility and dissolution. Asian Journal of Biomedical and Pharmaceutical Sciences 2012; 15: 7-14.

10. Shafiq S, Shakeel F, Talegaonkar S, et al. Development and bioavailability assessment of ramipril nanoemulsion formulation. Eur J Pharm Biopharm 2007; 66: 227-243.

11. Singh AK, Chaurasiya A, Singh M, et al. Exemestane Loaded Self-Microemulsifying Drug Delivery System (SMEDDS): Development and Optimization", AAPS Pharm Sci Tech 2008; 9: 628-634.

12. Subramanian N, Ray S, Ghosal SK, et al. Formulation Design of SelfMicroemulsifying Drug Delivery Systems for Improved Oral Bioavailability of Celecoxib. Biol Pharm Bull 2004; 27: 1993-1999.

13. Balakrishnan $\mathrm{P}$, Lee $\mathrm{BJ}$, Oh $\mathrm{DH}$ et al. Enhanced oral bioavailability of coenzyme Q10 by a novel solid self-emulsifying drug delivery system. Int J Pharm Sci 2009; 374: 66-72.

14. Zhao Y, Wang C, Chow AH. Selfnanoemulsifying drug delivery system (SNEDDS) for oral delivery of Zedory Essential oil: Formulation and bio availability studies. Int J Pharm 2010; 383: 170-177.

15. Rokad V, Nagda C, Nagda D. Design and evaluation of solid self-emulsifying drug delivery system of rosuvastatin calcium. J Young Pharm 2014; 6: 37-45.

16. Kim JY, Young SK. Enhanced absorption of Indomethacin after oral or rectal administration of Self emulsifying system containing Indomethacin torats. Int J Pharm 2000; 194: 81-89.

17. Kouchak M, Avadi MR, Abbaspour MR, et al. Effect of different molecular weights of chitosan on preparation and Characterization of insulin loaded nanoparticles by ion gelation method. Int $\mathbf{J}$ Drug Dev \& Res 2012; 4: 271-277. 
18. Puttachari S, Kalyanea NV, Gupta SD. Design and evaluation of self-micro emulsifying drug delivery systems of acyclovir. Int J Pharm Pharm Sci 2014; 6: 677-681.

19. Harshal DM, Tanvir S, Dheeraj B, et al. Design and development of Solid micro emulsifying drug delivery system (SMEDDS) of Fenofibrate. Int $\mathrm{J}$ Pharm Pharm Sci 2011; 3: 163-166.

20. Bachhav YG, Patravale. VB. SMEDDS of Glyburide: Formulation, In Vitro Evaluation, and Stability Studies. AAPS Pharm SciTech 2009; 10: 482-487.

21. Patil PP, Vaishali K, Santosh P. Potential Investigation of Peceol for formulation of Ezetimibe self nano emulsifying Drug Delivery Systems. Asian Journal of Biomedical and Pharmaceutical Sciences 2016; 6: 21-32.

22. Eccleston J. Microemulsions; In: Swarbrick J \& Boylan JC. (eds.) Encyclopedia of
Pharmaceutical Technology. New York, USA: Marcel Dekker 1999.

23. Sullivan JR, Gad SC, Julien M. A review of the nonclinical safety of Transcutol ${ }^{\circledR}$, a highly-purified form of diethylene glycol monoethyl ether (DEGEE) used as a pharmaceutical excipient. Food Chem Toxicol 2014; 72: 40-50.

24. Lawrence MJ, Rees GD. Microemulsionbased media as novel drug delivery systems. Adv Drug Deliv Rev 2000; 45: 89121.

25. Chu KS, Schorzman AN, Finniss MC, et al. Nanoparticle drug loading as a design parameter to improve docetaxel pharmacokinetics and efficacy. Biomaterials 2013; 34: 1-13.

26. Yang SC, Benita S. Enhanced absorption and drug targeting by positively charged submicron emulsions. Drug Dev Res 2000; 50: 476-486.

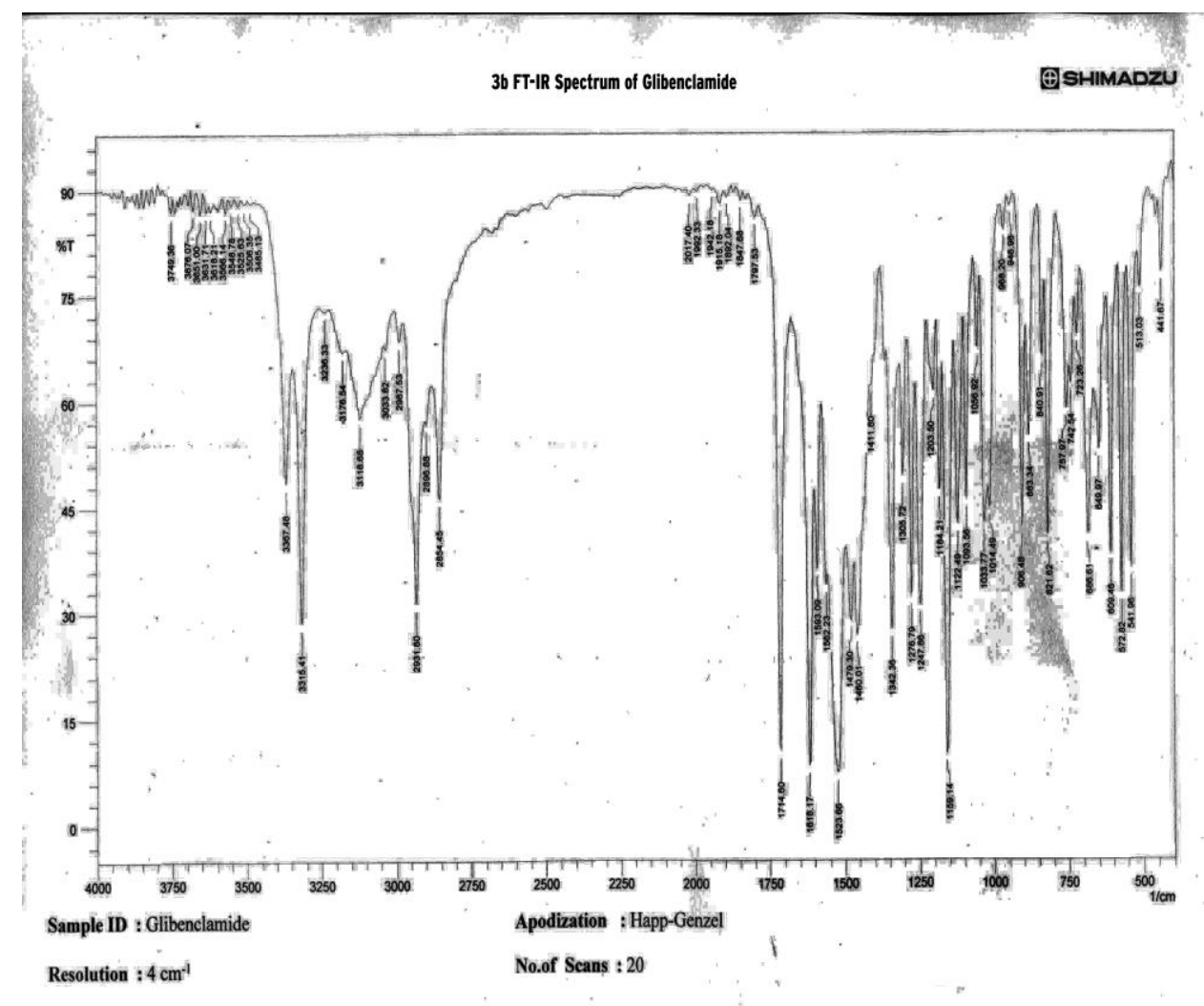

Figure 1: FT-IR spectrum of glibenclamide 


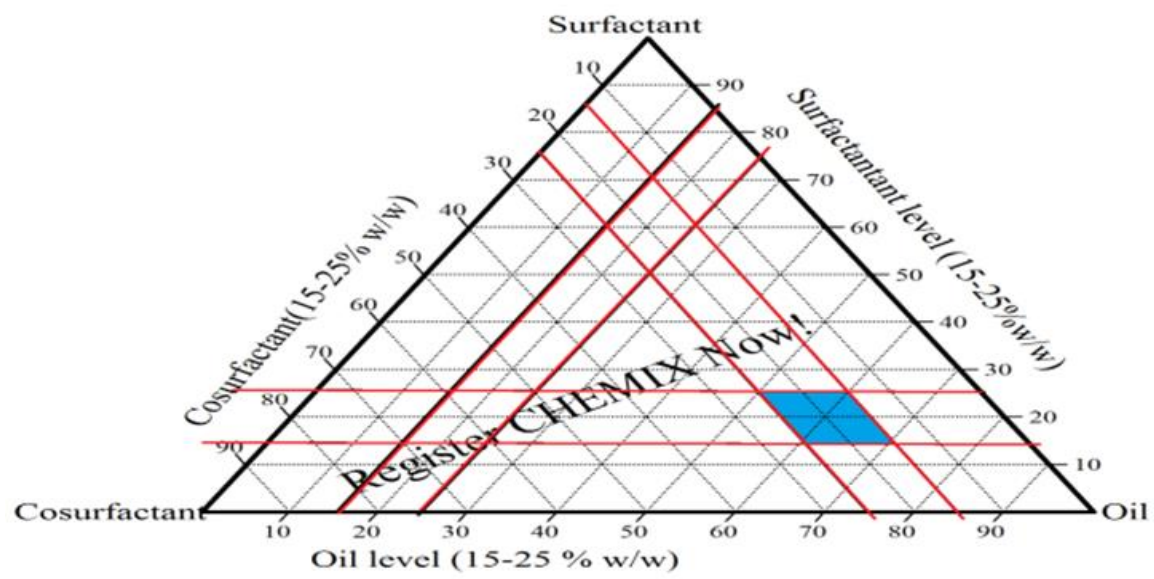

Figure 2: Ternary phase diagram of glibenclamide SEDDS

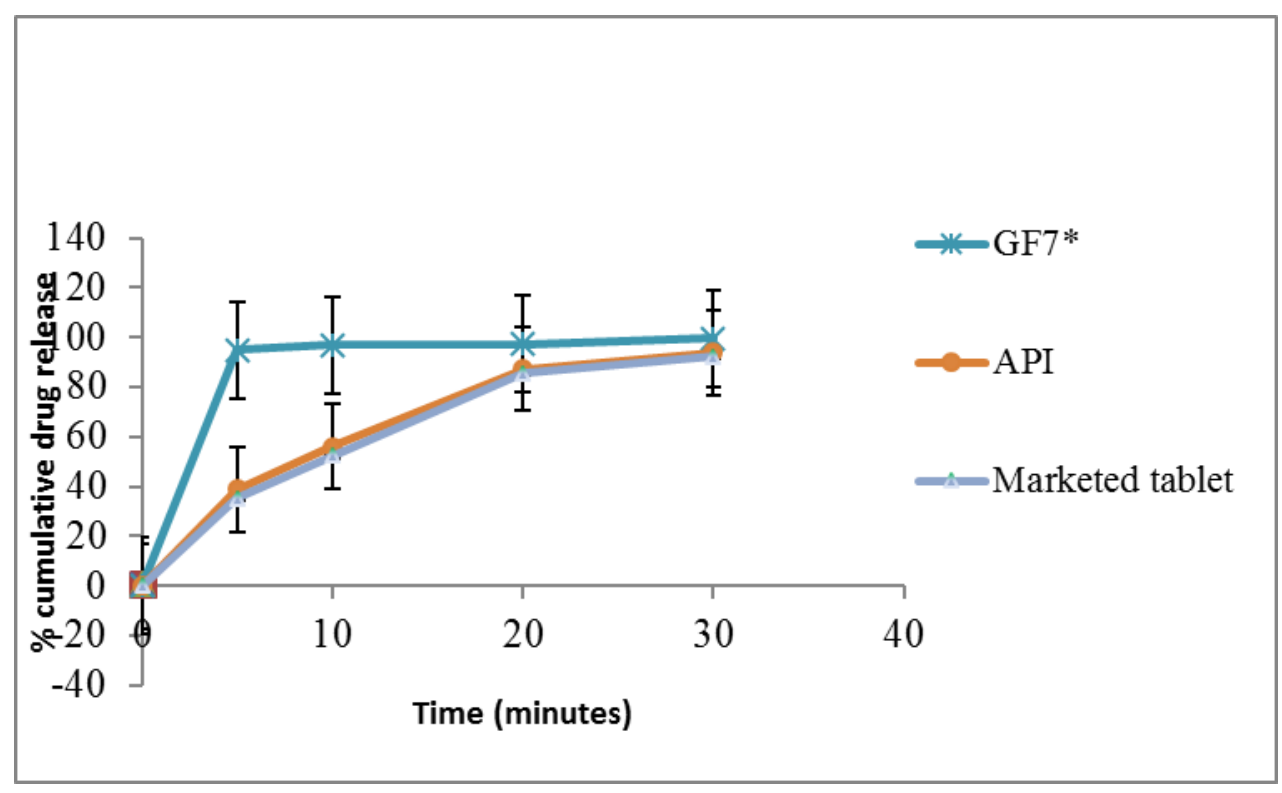

Figure 3: Dissolution comparison graph of API, marketed formulation and optimized formulation of glibenclamide SEDDS

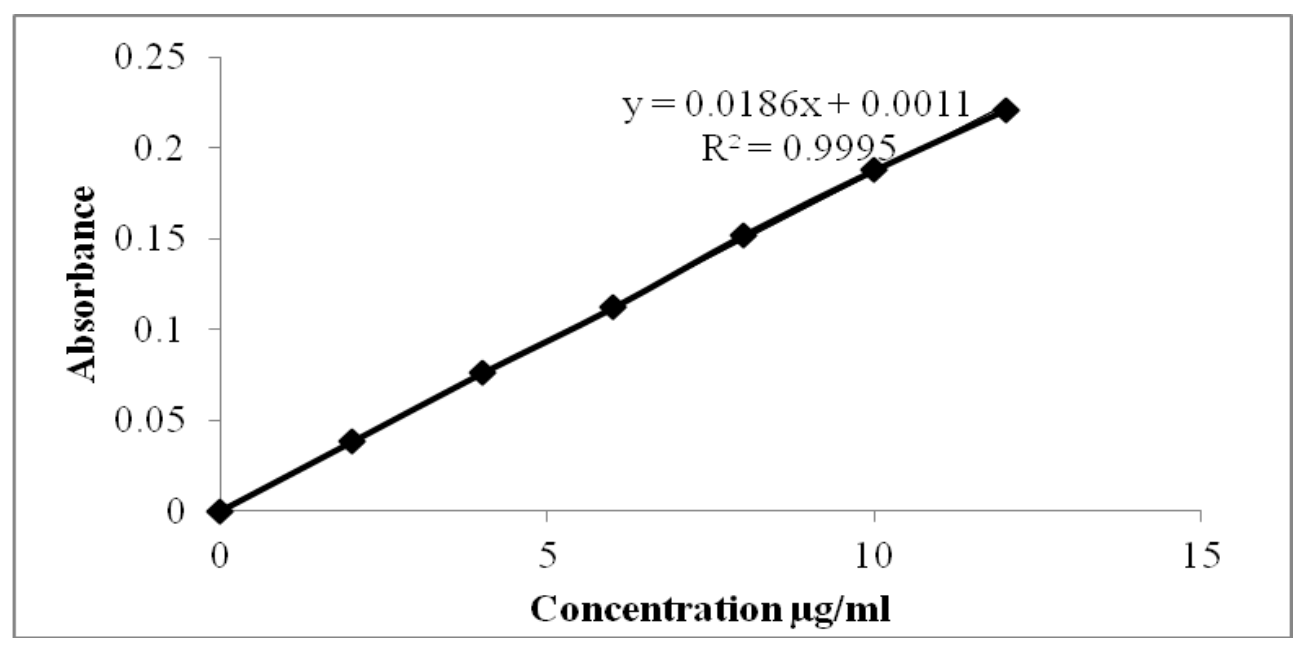

Figure 4: Calibration curve of glibenclamide in phosphate buffer pH 7.4

Table 1: Solubility of glibenclamide in various excipients 


\begin{tabular}{|c|l|c|}
\hline S.No. & \multicolumn{1}{|c|}{ Excipients } & Glibenclamide Solubility $(\mathbf{m g} / \mathbf{m l})$ \\
\hline 1. & Virgin sesame oil & $3.5 \pm 0.012$ \\
\hline 2. & Virgin coconut oil & $6.30 \pm 0.007$ \\
\hline 3. & Sunflower oil & $2.28 \pm 0.001$ \\
\hline 4. & Corn oil & $4.89 \pm 0.009$ \\
\hline 5. & Mustard oil & $2.34 \pm 0.002$ \\
\hline 6. & Rice bran oil & $4.25 \pm 0.001$ \\
\hline 7. & Olive oil & $5.65 \pm 0.003$ \\
\hline 8. & Peceol & $7.83 \pm 0.015$ \\
\hline 9. & Labrasol & $9.52 \pm 0.016$ \\
\hline 10. & Labrafil 1944CS & $6.24 \pm 0.004$ \\
\hline 11. & Labrafil 2125 & $1.26 \pm 0.011$ \\
\hline 12 & Capryol PGMC & $2.26 \pm 0.052$ \\
\hline 13. & Transcutol HP & $18.12 \pm 0.018$ \\
\hline 14. & Distilled water & Solvents \\
\hline 21. & Dichloromethane & $0.0001 \pm 0.008$ \\
\hline $15 .$. & Ethanol & $0.0092 \pm 0.002$ \\
\hline
\end{tabular}

Table 2: Composition of glibenclamide SEDDS formulations

\begin{tabular}{|c|c|c|c|c|}
\hline Formulation Code & Oil (mg) & Smix (mg) & Particle size (nm) & Drug Loading (\%) \\
\hline GF1 & 15 & 40 & 222.2 & $92.3 \pm 3.26$ \\
\hline GF2 & 15 & 30 & 284.2 & $88.8 \pm 2.38$ \\
\hline GF3 & 25 & 30 & 415.2 & $82 \pm 1.9$ \\
\hline GF4 & 25 & 40 & 233.1 & $84 \pm 4$ \\
\hline GF5 & 20 & 40 & 402.3 & $81.2 \pm 5.4$ \\
\hline GF6 & 25 & 50 & 229.7 & $89 \pm 4.16$ \\
\hline GF7* & 20 & 40 & 421.6 & $85.6 \pm 2.64$ \\
\hline GF8 & 20 & 30 & 464.1 & $92.3 \pm 4.12$ \\
\hline GF9 & 20 & 50 & 616.3 & \\
\hline
\end{tabular}


Table 3: Self- emulsification and drug precipitation of glibenclamide SEDDS

\begin{tabular}{|c|c|c|c|}
\hline Formulation Code (FC) & Visibility grade & Phase separation & Precipitation \\
\hline GF1 & IV & + & ++ \\
\hline GF2 & III & + & ++ \\
\hline GF3 & IV & + & ++ \\
\hline GF4 & V & X & ++ \\
\hline GF5 & II & + & ++ \\
\hline GF6 & III & X & XX \\
\hline GF7 $*$ & I & + & ++ \\
\hline GF8 & V & + & ++ \\
\hline GF9 & III & + & \\
\hline
\end{tabular}

$X$-No phase separation, $X X$-No precipitation, +-phase separation and ++-precipitation.

Table 4: Refractive index, Turbidity, Optical clarity, Polydispersity index (PDI), Viscosity, Cloud point measurement and Emulsification time of SEDDS formulations of glibenclamide

\begin{tabular}{|c|c|c|c|c|c|c|c|}
\hline FC & $\begin{array}{l}\text { Refractive } \\
\text { Index } \\
\pm \text { SD }(n=3)\end{array}$ & $\begin{array}{l}\text { Turbidity } \\
\text { (NTU) }\end{array}$ & $\begin{array}{l}\text { Absorb } \\
\text { ance }\end{array}$ & $\begin{array}{l}\text { PDI } \\
\pm \text { SD } \\
(n=3)\end{array}$ & $\begin{array}{l}\text { Viscosity } \\
(\text { cps }) \\
\pm \quad \text { SD } \\
(n=3)\end{array}$ & $\begin{array}{l}\text { Cloud point } \\
\text { measurement } \\
\left({ }^{\circ} \mathrm{C}\right) \\
\pm \mathrm{SD}(\mathrm{n}=3)\end{array}$ & $\begin{array}{l}\text { Emulsification } \\
\text { time (sec) }\end{array}$ \\
\hline GF1 & $\begin{array}{c}1.3333 \pm \\
0.0006\end{array}$ & 98 & 0.583 & $\begin{array}{c}0.204 \\
\pm \\
0.017\end{array}$ & $211 \pm 2.22$ & $68 \pm 2.16$ & 23 \\
\hline GF2 & $\begin{array}{c}1.3332 \pm \\
0.0003\end{array}$ & 93 & 0.578 & $\begin{array}{r}0.315 \\
\pm 0.21\end{array}$ & $215 \pm 2.36$ & $69 \pm 3.61$ & 32 \\
\hline GF3 & $\begin{array}{c}1.3356 \pm \\
0.0005\end{array}$ & 127 & 0.598 & $\begin{array}{l}0.2 \pm \\
0.01\end{array}$ & $324 \pm 3.24$ & $72 \pm 2.23$ & 24 \\
\hline GF4 & $\begin{array}{c}1.3331 \pm \\
0.0002\end{array}$ & 134 & 0.488 & $\begin{array}{r}0.284 \\
\pm 0.01\end{array}$ & $356 \pm 1.72$ & $77 \pm 2.46$ & 25 \\
\hline GF5 & $\begin{array}{c}1.3334 \pm \\
0.0002\end{array}$ & 142 & 0.571 & $\begin{array}{r}0.438 \\
\pm 0.01\end{array}$ & $318 \pm 2.34$ & $71 \pm 2.76$ & 27 \\
\hline GF6 & $\begin{array}{c}1.3335 \pm \\
0.0003\end{array}$ & 132 & 0.472 & $\begin{array}{r}0.224 \\
\pm 0.02\end{array}$ & $365 \pm 3.24$ & $79 \pm 4.20$ & 20 \\
\hline GF7* & $\begin{array}{c}1.3330 \pm \\
0.0002\end{array}$ & 92 & 0.494 & $\begin{array}{r}0.244 \\
\pm 0.04\end{array}$ & $200 \pm 1.95$ & $62 \pm 2.25$ & 21 \\
\hline
\end{tabular}




\begin{tabular}{|c|c|c|c|c|c|c|c|}
\hline GF8 & $1.3358 \pm$ & 125 & 0.687 & 0.423 & $348 \pm 1.89$ & $73 \pm 2.36$ & 30 \\
& 0.0004 & & & \pm 0.06 & & & \\
\hline GF9 & $1.3349 \pm$ & 132 & 0.708 & 0.328 & $298 \pm 2.97$ & $78 \pm 4.28$ & 22 \\
& 0.0004 & & & \pm & & & \\
& & & & 0.015 & & & \\
\hline
\end{tabular}

Table 5: Cumulative percent release of glibenclamide SEDDS from various formulations

\begin{tabular}{|c|c|c|c|}
\hline Time in min & GF7* SEDDS & API & Marketed Tablet \\
\hline 0 & 0 & 0 & 0 \\
\hline 5 & $95 \pm 3.51$ & $38.7 \pm 1.98$ & $35.31 \pm 2.22$ \\
\hline 10 & $96.8 \pm 1.97$ & $55.9 \pm 2.24$ & $52.4 \pm 3.71$ \\
\hline 20 & $97.2 \pm 3.14$ & $87.3 \pm 3.21$ & $85.5 \pm 3.26$ \\
\hline 30 & $99.7 \pm 2.74$ & $93.8 \pm 2.67$ & $92.3 \pm 3.47$ \\
\hline
\end{tabular}

Table 6: Percent cumulative drug absorbed through dialysis membrane of optimized glibenclamide SEDDS formulations

\begin{tabular}{|c|c|c|}
\hline Time in hours & GF7* SEDDS & Marketed Tablet \\
\hline 0 & 0 & 0 \\
\hline 0.5 & $97.2 \pm 0.95$ & $82.92 \pm 1.97$ \\
\hline 1 & $99.3 \pm 1.74$ & $90.32 \pm 2.19$ \\
\hline 2 & $99.8 \pm 2.12$ & $96.23 \pm 1.41$ \\
\hline
\end{tabular}


This manuscript was peer-reviewed

Mode of Review: Single-blinded

Editor: Mr. Mirza Salman Baig

International Journal of Pharmaceutics and Pharmacology is an open access, peer reviewed journal published by Edwiser International.

Submit your valuable manuscript at-

editor.ijpp@edwiserinternational.com

submit.manuscript@edwiserinternational.com

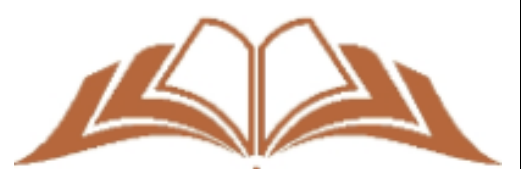

EDW $\mathrm{D}$ S E R
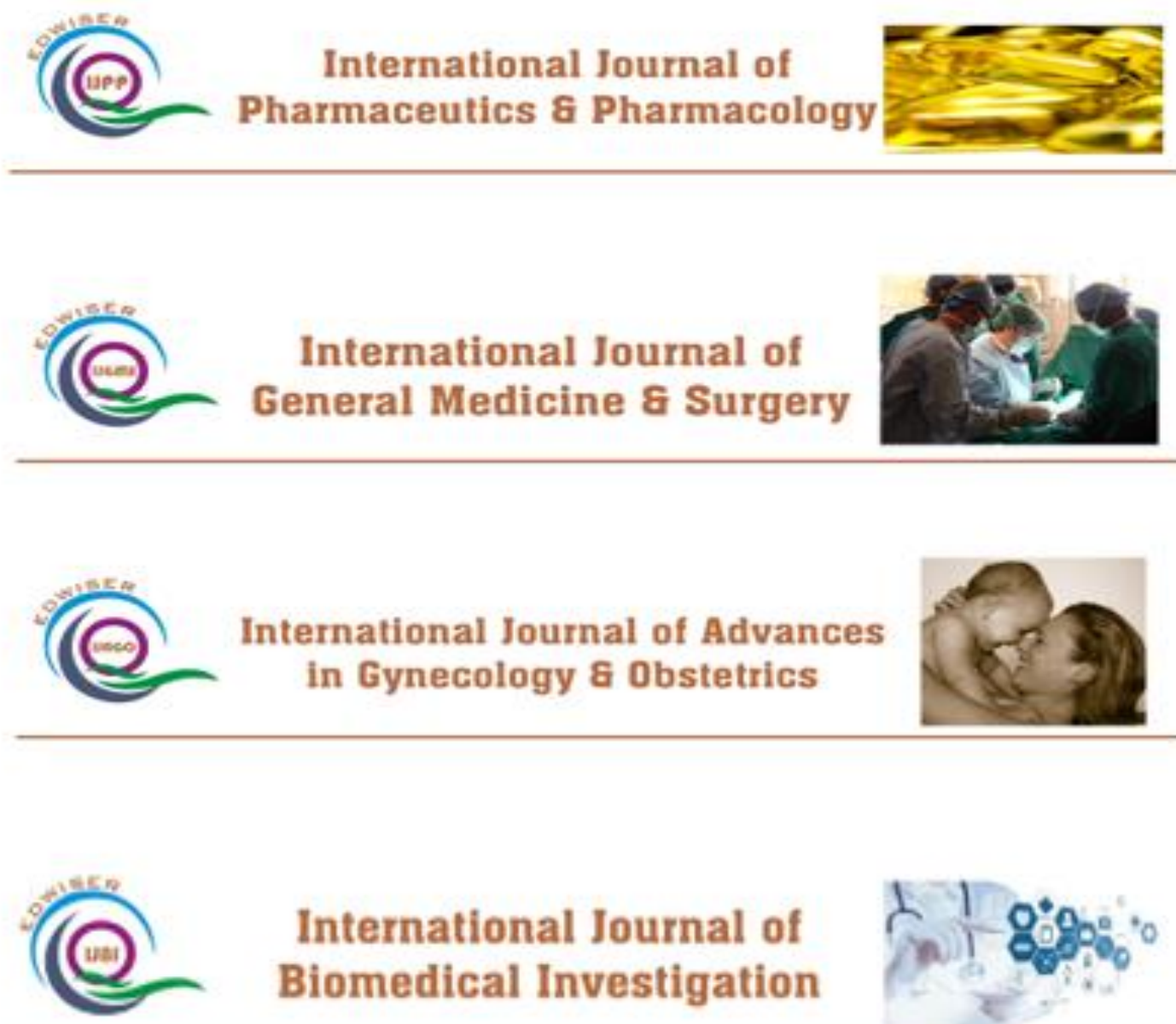

\section{International Journal of Biomedical Investigation}
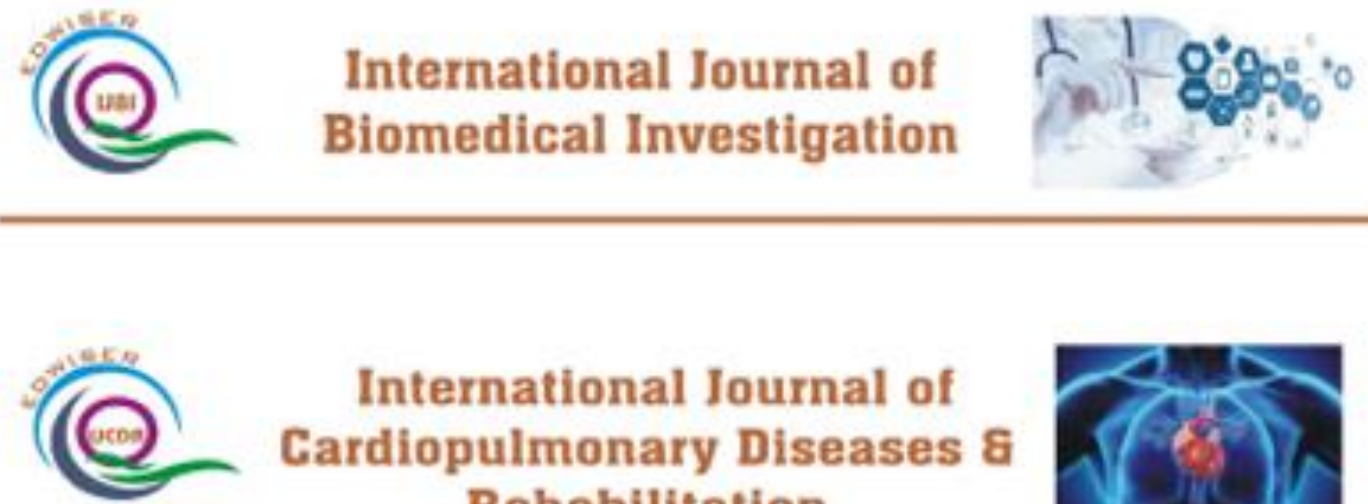

\section{International Journal of Cardiopulmonary Diseases \& Rehabilitation}

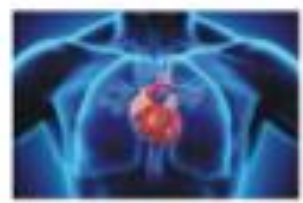

